\title{
Use of Impella cardiac axial flow pump for cardiogenic shock (A newer alternative)-How good is the evidence?
}

\author{
Rafiq Ahmed BHAT ${ }^{1}$; Syed Manzoor Ali ${ }^{2}$; Yoosuf Ali Ashraf Muhammad HUSSENbOCUS ${ }^{3}$; Akanksha RATHI ${ }^{4}$; \\ Javaid Akhter BHAT ${ }^{5}$; Abdul Aleem $\mathrm{KHAN}^{6}$; Syed MAQBOOL ${ }^{2}$; Raja SAQIB IQBAL ${ }^{7}$; Md Monowarul ISLAM ${ }^{8}$;

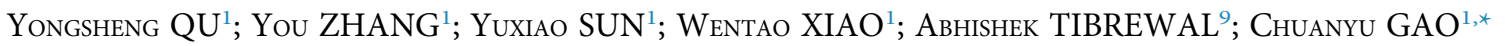 \\ ${ }^{1}$ Zhengzhou University People's Hospital, Department of Cardiology, Fuwai Central China Cardiovascular Hospital, Zhengzhou, China \\ 2 Department of Cardiology, Super Speciality Division, Government Medical College, Srinagar, India \\ ${ }^{3}$ Department of Rheumatology and Immunology, Nanjing Drum Tower Hospital Clinical College of Nanjing Medical University, Nanjing, China \\ ${ }^{4}$ Department of Community Medicine, Vedanta Institute of Medical Sciences, Palghar, India \\ ${ }^{5}$ State Key Laboratory of Crop Genetics and Germplasm Enhancement, Nanjing Agricultural University, Nanjing, China \\ ${ }^{6}$ Department of Orthopaedics, Nanjing First Hospital, Nanjing Medical University, Nanjing, China \\ 7 Department of Paediatrics, Batra Hospital and Medical Research Centre, New Delhi, India \\ ${ }^{8}$ Zhengzhou University First Affiliated Hospital, Zhengzhou, China \\ 9 Department of Community Medicine, Institute of Biostatistics and Epidemiology, Gurgaon, India
}

Key words: Cardiogenic shock, Hemodynamic support, Impella Cardiac Axial Pump, IABP, VA-ECMO

\begin{abstract}
The adverse outcomes of a ventricular heart failure (left, right or biventricular) caused by cardiogenic shock are aggravated by lung oedema and organ mal perfusion. Despite advances in medical sciences, revascularisation and mechanical hemodynamic support have proved ineffective in reducing the mortality rate in such patients. A thorough study of the data available about cardio-vascular diseases reveals that the application of conventional methods of treatment are least helpful to practically restore normal functions of heart when it experiences end-stage systolic ventricular failure. Thus, to overcome the challenges and find alternatives to address this issue, percutaneous ventricular support devices/machines were designed and successfully introduced. These devices have revolutionized the treatment of ventricular heart failures and are now in use all over the world. In this review paper a newer mechanical circulatory support (MCS) device, Impella, has been discussed and compared with a few other devices like (Intra-aortic Balloon Pump (IABP), Extracorporeal Circulation (ECLS) and Veno-arterial Extracorporeal Membrane Oxygenation (VA-ECMO). This article studies the challenges being faced during the treatment of cardiogenic shock, and thoroughly discusses the use and effectiveness of Impella Cardiac Axial Pump in each emergency. It can be said that mechanical circulatory support (MCS) device use during percutaneous coronary intervention (PCI) should be individualized based on multiple factors with a recommended use in patients with the greatest potential benefit and a relatively low risk of device-related complications. The current literature suggests that the outcomes of use of Impella and other mechanical circulatory support devices like IABP and VA-ECMO are comparable. Though there seem to be a few advantages of Impella over the others, sufficiently powered, multi-centric, randomised control trials are needed to establish its superiority.
\end{abstract}

\section{Introduction}

Cardiogenic shock (CS) is defined as the state of critical end organ hypoperfusion, secondary to cardiac malfunction, as in the case of an acute myocardial infarction (AMI) (Mebazaa et al., 2018; Ibanez et al., 2018; van Diepen et al., 2017). The

*Address correspondence to: Chuanyu Gao, gaocy6802@163.com Received: 30 March 2021; Accepted: 13 August 2021 traditional management of cardiogenic shock involves early diagnosis and improvement of tissue perfusion by optimising oxygen delivery (Hajjar and Teboul, 2019). Cardiogenic shock remains a challenging condition with mortality rates of around 50\% (Mandawat and Rao, 2017). To achieve a breakthrough in reducing mortalities and give a new lease of life to patients suffering from Acute Myocardial Infarction with cardiogenic shock (AMICS), percutaneous ventricular support devices have been introduced and successfully used 
to augment the treatment. However, mechanical support for reducing the effects of the shock should follow the medical therapy, which includes early detection, timely pharmacological intervention, and adequate respiratory support. Nevertheless, several long-term as well as short-term devices are now available for this purpose. Whereas long-term support device needs an open-heart surgery and requires long-term care, a short-term support device widely used in emergencies is connected through percutaneous membrane. We will be discussing about Impella, that is a newer Left Ventricular Assist Device (LVAD), in this review paper and will be comparing its outcome with that of a few other Mechanical Assist Devices for the heart, like Intra-aortic Balloon Pump (IABP), Extracorporeal Life Support (ECLS) and Veno-arterial Extracorporeal Membrane Oxygenation (VA-ECMO). Discussing other heart assist devices is outside the scope of this paper.

\section{Mechanical Circulatory Support (MCS) Devices}

After the discovery of cardiopulmonary bypass and open-heart surgery in 1950s, an interest in mechanical circulatory support (MCS) also developed concurrently. They can either be long term devices or short-term devices depending on the duration of the support that is expected from them. While the long-term devices need an open-heart surgery, a shortterm device can be implanted percutaneously in an AMI patient to provide support for the time till a percutaneous coronary intervention (PCI) is done to improve the heart function. This is often called bridge to transplantation (BTT). An overview of the temporary MCS devices is given in Table 1.

\section{Intra-Aortic Balloon Pump (IABP)}

As per the guidelines published by the European Society of Cardiology (ESC) in 2017, it has been suggested that mechanical circulatory support in patients presented with Acute Myocardial Infarction, with ST-Segment elevation, may be considered when therapy with a vasopressor and inotrope is inadequate. Such devices are being widely used during AMICS for decades now, however, with limited effectiveness. Of these devices Intra-Aortic Balloon Pumps (IABPs), for example, are in wide use globally as mechanical circulatory support during an Acute Myocardial Infarction cardiogenic shock. This device consists of a cylindrical polyurethane balloon that is placed in the aorta, about $2 \mathrm{~cm}$ (0.79 in) from the left subclavian artery (Khan and Siddiqui, 2020). The balloon inflates and deflates through the process of counter pulsation, implying that it deflates in systole and inflates in diastole. During Systolic deflation period, a significant reduction in afterload can be seen through the vacuum effect, while the forward flow from the heart increases indirectly. Diastolic inflation increases blood flow to the coronary arteries via retrograde flow. These two fundamental functions working in unison reduce myocardial oxygen demand and increase its supply. But these devices failed to yield desired results vis-à-vis their effectiveness in arresting the mortality rate in patients during IABPSHOCK II trial and hence were downgraded by ESC. Correspondingly, short-term devices, too, have proved a failure in saving lives of patients under cardiogenic shock.

\section{Complications and contraindications of IABP}

IABP can cause thrombocytopenia, when its membrane interacts with platelets or due to heparinization when the device is in use. However, it does not lead to any severe adverse consequences. Other complications associated with IABP include fever, vascular injury (including aortic dissection), thromboembolism, limb ischemia, bleeding, device retention/entrapment, and balloon rupture. Vascular injury to the femoral artery may need repair at the time of removal of an IABP device, hence vital arrangements should be in place while removing them. Amongst some major contraindications of IABP use, as moderate or worse aortic insufficiency, aortic dissection, aortic aneurysm, uncontrolled coagulopathy, and severe sepsis are fully established and well known. It has been observed that aortic arch can cause a blockage of renal artery, leading to renal failure if the balloon is placed away from it. Other possible complications include cerebral embolism during insertion, infection, dissection of the aorta or iliac artery, perforation of the artery and bleeding in the mediastinum. The balloon is prone to mechanical failure, and vascular surgery is the only way to remove it. Even after removing the balloon there is a risk of 'embolic shower' from micro clots that may have formed on the surface of the balloon, leading to peripheral thrombosis, myocardial ischemia, hemodynamic decompensation, and late

TABLE 1

Technical properties of the temporary MCS Devices

\begin{tabular}{lllll}
\hline Parameters & ECLS & Impella & IABP & ECMO \\
\hline Insertion & Percutaneous & Percutaneous, arterial access & Percutaneous & Percutaneous \\
Max flow (L/min) & 7.0 & $2.5-5.5$ & - & $4.0-7.0$ \\
Circulatory support (\%) & $75-100$ & $30-100$ & 15 & - \\
Anticoagulation & + & + & + & + \\
Peripheral Vascular Injury & Low & Moderate & Very low & High \\
Pump mechanism & Centrifugal & Axial & Pneumatic & Centrifugal \\
Recommended duration of use & 7 days & 10 days & Days-weeks & $7-10$ days \\
Risk of hemolysis & Low & Low & Very low & Moderate \\
\hline
\end{tabular}

Note: ECLS, extracorporeal life support; IABP, intra-aortic balloon pump. Source: Cardozo et al. (2015). 
pseudoaneurysm. Relative contraindications include any contraindications to anticoagulation, which is generally required to use the device safely; significant peripheral arterial disease at the access site that makes safe implant of the device difficult, and LV outflow tract obstruction, which could worsen with IABP use due to the functional after-load reduction of the device. IABP is also not very effective if the LV is dysfunctional (Sintek et al., 2015; Patel et al., 2011; Kapur et al., 2015).

\section{Extracorporeal Life Support (ECLS) \& VA-ECMO}

Out of the most commonly used temporary Mechanical circulatory Support (MCS) devices, is Extracorporeal Life Support (ECLS), which is far less expensive than other devices sold in the market. The plus point is that it is readily available in all major hospitals or super specialty medical centres worldwide, where facilities are in place for acute percutaneous coronary intervention (PCI) procedures. The device can be installed by the bedside of a patient. Its suitability for use during a cardiopulmonary bypass, as an alternative to the heart-lung-machine, makes ECLS an attractive option for circulatory support (Nersesian et al., 2019). ECLS provides artificial support to the functioning of lung and/or heart, as it helps maintain constant oxygenation of a patient's blood outside his body and returns it back into the body with the help of a pump, thus giving the heart and lungs much needed rest. This machine works on a circuitry system that controls a series of tubes through which blood flows to an artificial lung, which functions in the same way as natural lungs do; giving oxygen to the blood and extracting carbon dioxide out of it. This oxygenated blood then is pumped back to the body through the same ECLS circuitry, with the help of an inbuilt specialized pump.

There are two types of ECLSs, which are Veno-arterial (VA) and Veno-Venous. Veno-arterial ECLS takes out blood from the vein and pumps it into the artery under high pressure to help the pumping function of a failing heart. Veno-Venous (VV) ECLS is used solely to help lung function, which involves removal and return of blood through the veins, usually near the heart. New innovations in designing have been made to this support system during the past few decades. Modern machines are lightweight, which can be carried by a person or transported in an ambulance or helicopter.

The first type of ECLS devices, also known VA-ECMO, are generally used-

1. To provide backup to medical therapy during heart and/or lung failure and help them to recover

2. To function as a bridge for further treatment such as heart assist device or left ventricular assist device (LVAD)

3. As a bridge during heart or lung transplantation

Despite its multidimensional functions VA-ECMO has proved least effective in unloading the ventricle. To the contrary, however, it causes high afterload due to outflow in the aorta, which in turn leads to an increase in demand for more oxygen intake, and myocardial wall tension, resulting in worsening coronary perfusion. Besides, blood stasis in the left ventricle leads to pulmonary hypertension and interstitial lung oedema. There are some options to improve ventricular unloading though, starting with inotropic support and escalating to intra-aortic balloon pump (IABP) (Nersesian et al., 2019).

\section{Impella}

\section{Device characteristics}

Of all the known life support devices that are in use in the field of cardiovascular diseases, Impella (Abiomed, Danvers, Massachusetts, USA) is a highly potential substitute for percutaneous mechanical circulatory support (pMCS) which is being used all over the world today as a path to recovery. Impella is based on a simple screw pump invented by a Greek philosopher and mathematician Archimedes in the 3rd Century B. C. This pump is cylindrical, with helical column inside the cylinder. It is used to draw fluid from bottom to top by rotating the cylinder on its longitudinal axis. Impella device works on the same principle as does the Archimedean screw pump. The device consists of a small axial flow pump fitted onto a pigtail catheter. A cylinder in the device rotates on its longitudinal axis, pumping the fluid from the bottom of the column to the top. Impella is used in the same way to enhance cardiac output (Fig. 1).

Four (fifth one is experimental) different variants of the Impella devices are in use today, which are:

- Impella 2.5, with a cardiac output maximising capacity of up to $2.5 \mathrm{~L} / \mathrm{min}$

- Impella CP, with a cardiac output increasing capacity of up to $4.0 \mathrm{~L} / \mathrm{min}$

- Impella 5.0, with a cardiac output increasing capacity of up to $5.0 \mathrm{~L} / \mathrm{min}$, and

- Impella RP, designed for use in the RV

Of these four variants, the Impella 2.5 and Impella CP can be installed percutaneously, whereas the Impella 5.0 requires a cut down the femoral artery or axillary access for proper placement (Table 2). Apart from the artery access, a grafted vessel may also be constructed to keep the implant in place.

\section{Function and hemodynamic effects of Impella}

Impella device is implanted by entering the femoral artery with a 13-French sheath. The established procedure of implantation is to insert a guidewire and catheter into the aorta through the aortic valve and as far as Left Ventricle (LV). The catheter is then exchanged for the manufacturersupplied guide wire, and the device is loaded onto this wire and pushed until it crosses the aortic valve, in a fashion that the inlet portion of the device is in the LV and the outlet portion of the device in the ascending aorta (Fig. 2). Transoesophageal Echocardiography (TEE) and/or fluoroscopy can be used to assist in proper device placement. To confirm the placement site of Impella, its easier and less invasive to use trans-thoracic echocardiography rather than trans-oesophageal echocardiography. Additionally, the pressure waveform of the monitor can be used as a reference. The device is attached to a computer console, which can be used to control the device function. 


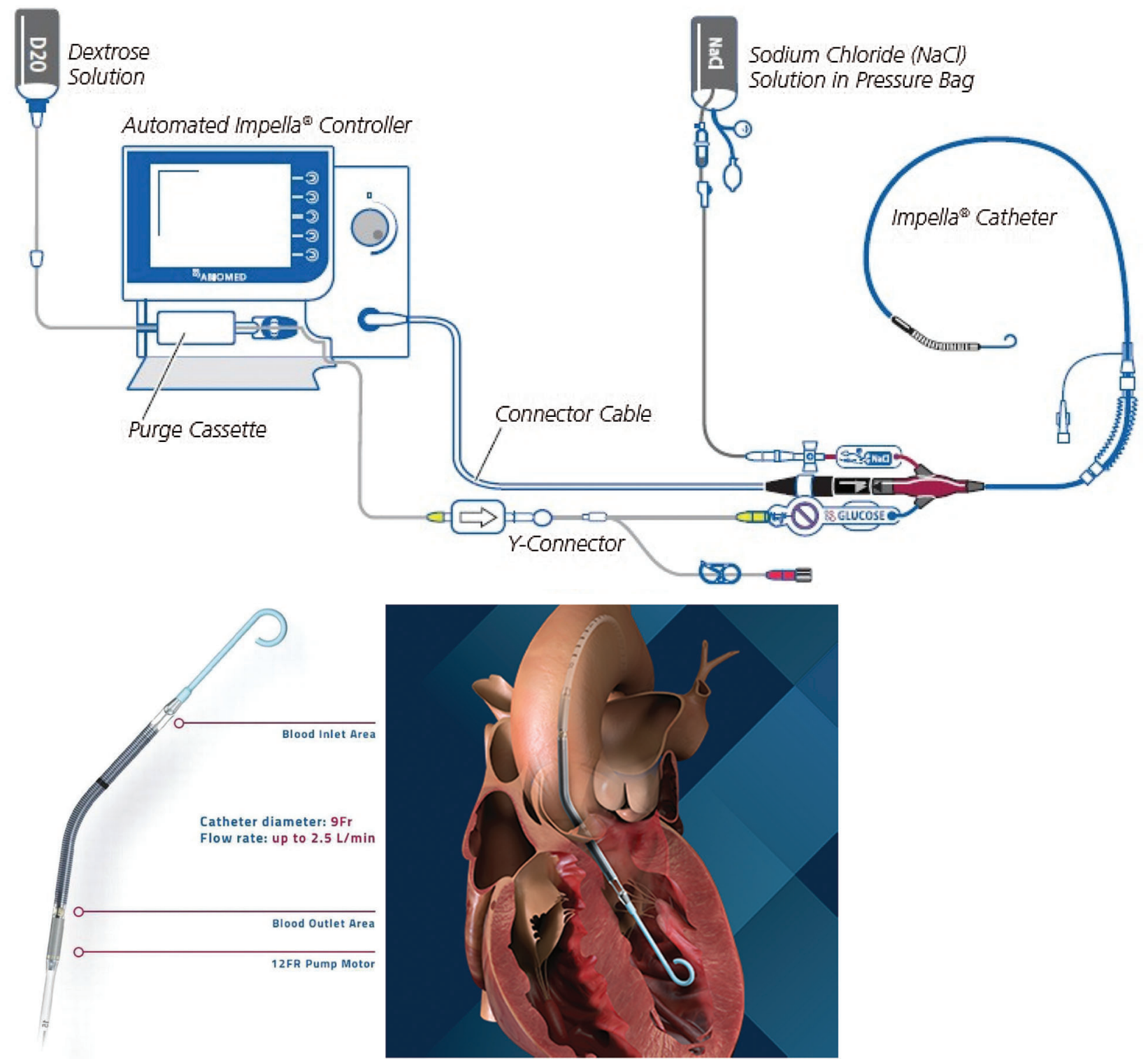

FIGURE 1. Standard configuration of the Automated Impella. Source: Health Jade Team (2019).

\section{TABLE 2}

Types of Impella devices

\begin{tabular}{llll}
\hline Characteristics & Impella 2.5 & Impella CP & Impella 5.0 \\
\hline Flow & $<2.5 \mathrm{~L} / \mathrm{min}$ & $<4.0 \mathrm{~L} / \mathrm{min}$ & $<5 \mathrm{~L} / \mathrm{min}$ \\
Catheter Size & $9 \mathrm{~F}$ & $9 \mathrm{~F}$ & $9 \mathrm{~F}$ \\
Pump Insertion Size & $12 \mathrm{~F}$ & $14 \mathrm{~F}$ & $21 \mathrm{~F}$ \\
Approval Duration & 4 days (US) & 4 days (US) & 6 days (US) \\
& 5 days (EU) & 5 days (EU) & 10 days (EU) \\
FDA Approval Indications & High risk PCI AMICS/PCCS & High risk PCI AMICS/PCCS & AMICS/PCCS \\
Insertion Sheath & $13 \mathrm{~cm}$ & $13 \mathrm{~cm} / 25 \mathrm{~cm}$ Peel-Away (Femoral artery) & $6 \mathrm{~cm}$ \\
& Peel-Away (Femoral artery) & & Peel-Away \\
& & & (Axillary/Femoral graft) \\
Valve Interaction & Smooth Cannula & Smooth Cannula & Smooth Cannula \\
\hline
\end{tabular}

Note: AMICS: Acute myocardial infarction with cardiogenic shock; PCI: Percutaneous coronary intervention; PCCS: Post-cardiotomy Cardiogenic Shock (Table reproduced from: Hill et al. (2019)). 


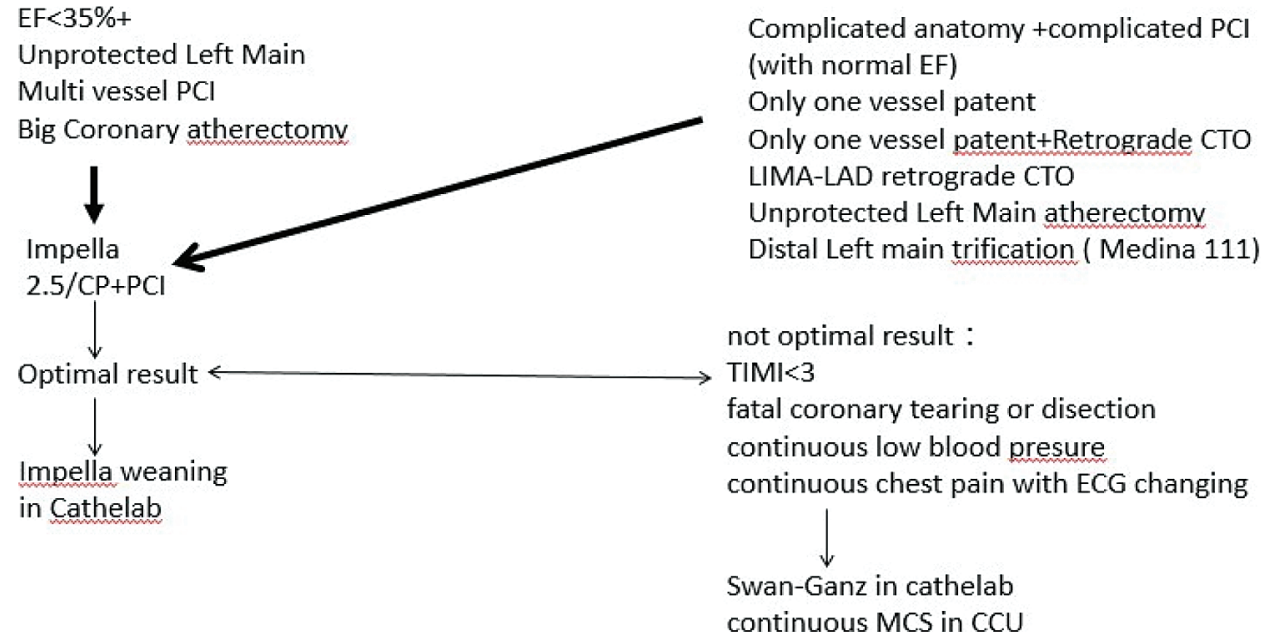

FIGURE 2. Flow chat of complicated PCI protected by Impella.
Rapid percutaneous induction of Impella is extremely important for patients with cardiogenic shock. Furthermore, if the increased auxiliary flow rate by Impella is effective in stabilizing hemodynamics, it may be effective to quickly upgrade from Impella $2.5 / \mathrm{CP}$ to Impella 5.0/5.5. The device once safely planted in its right place provides cardioprotection and hemodynamic support by significantly unloading the left ventricle during a stroke and helps reduce myocardial oxygen consumption. More so, Impella device plays a pivotal role in providing critical support to heart in patients with a Cardiogenic Shock by increasing the mean arterial pressure and cardiac output, besides relieving workload of the left ventricle and reduction of left ventricular wall stress, thereby reducing myocardial oxygen consumption, and improving myocardial perfusion. It prevents pulmonary oedema and involves use of very less anticoagulation than ECLS. A person who has suffered a cardiogenic shock fails to respond to conventional treatment measures, he needs supportive mechanical circulatory system to revive him. The Impella Ventricular Support System of the required model can easily complete the work. Impella device therapy is aims to provide adequate blood circulation (to replace or assist left ventricle pumping) whereas, at the same time, it provides the affected heart muscle an opportunity to rest and recuperate. The device may either be withdrawn (depending on the severity of damage to the heart), or maybe allowed to remain and provide a bridge to permanent means of circulatory aid. Impella has been approved for the treatment of recurrent or sudden episodes of cardiogenic shock occurring immediately (within $48 \mathrm{~h}$ ) after a heart attack or open-heart surgery, or in the event of acute left heart failure of an already diseased heart (cardiomyopathy), during pregnancy, postpartum, or as the result of myocarditis (a viral heart infection) (Health Jade Team, 2019; Fig. 3).

\section{Impella 5.5}

The Abiomed Impella 5.5 (Danvers, MA), is a newly developed axial flow trans-aortic cardiac support device mounted on a 9 Fr steering catheter with a 21 Fr pump cannula. During the 2019 Covid pandemic, emergency use of Impella 5.5 for critical care patients, who were suffering from COVID-19 complications undergoing ECMO treatment, was approved by the United States food and drug administration (FDA) under emergency use authorization (EUA). The first 1,000 patients have been treated primarily for cardiomyopathy, AMI cardiogenic shock, and postcardiotomy cardiogenic shock, with an average duration of support of 14 days. More implantations are needed to make any speculations about this device, but the high flow rate promises rapid hemodynamic restoration in patients if timely intervention is done (Bernhardt et al., 2020).

\section{Complications and contraindications of Impella}

The use of Impella for treatment has its own hazards. So, it is imperative that every possible precaution should be taken to avoid or minimise the damage from happening. This is important to see that the device is being placed properly to avoid possible complications like vascular injury or thrombosis, which may lead to cerebrovascular accident or limb ischemia, bleeding, coagulopathy, and injury to the aortic valve (Mebazaa et al., 2018; Mandawat and Rao, 2017). To lower the rate of vascular complications the angiographic- and fluoroscopic-guided vascular access maybe mandated (Johannsen et al., 2019). There are other complications, as well, which may be taken care of during the process. For example, left ventricular volume may be overloaded or heart function may be impaired. Heparinrelated complications may also occur. Regular repositioning of the device with TEE may be needed. The device is mechanical in nature and hence prone to malfunction. As with any invasive or intracardiac device, wrong placement can result in irrepressible injury or a disaster (Cardozo et al., 2015). Haemolytic anaemia secondary to Impella implantation is one of the documented complications (Cardozo et al., 2015). However, cases of severe haemolytic anaemia are rare.

Known contraindications of treatment with an Impella device include a prosthetic aortic valve, severely calcified aortic valve, Grade 2 or greater aortic insufficiency, and/or severe peripheral arterial disease. Relative contraindications include aortic disease, such as aortic dissection or aneurysm, or the presence of femoral-bypass grafts, which may be injured during device placement. Therefore, it is recommended to consult a vascular surgeon before starting the operation, especially if the patient has a parafetal aortic 


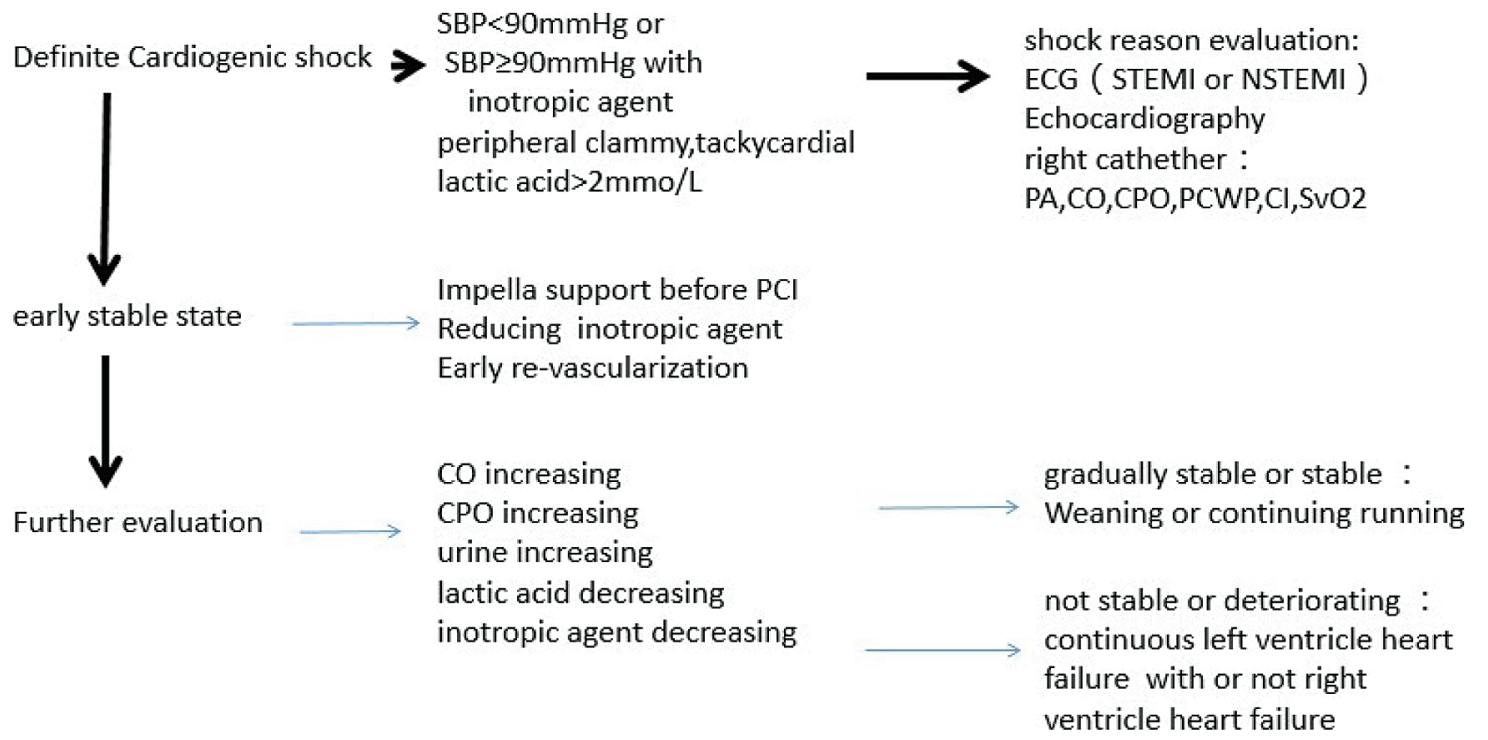

FIGURE 3. Cardiogenic shock impella application flow chat.

bypass graft at other access sites (such as subclavian or axillary artery). Probably, if the complications associated with Impella can be reduced, its effectiveness may be demonstrated, so it is also necessary to devise ways to reduce the complications.

\section{Comparison of MCS Devices and Their Role in LV Unloading}

Cardiogenic shock (CS) is developed because of a cascade of events that follows heart failure (HF). Heart failure leads to progressive decline in ventricular function as compensatory remodelling ultimately fails and patients present with recurrent episodes of acutely decompensated $\mathrm{HF}$ and ultimately CS (Esposito and Kapur, 2017). CS is characterised by impaired cardiac output leading to reduction in systemic perfusion, increased residual volume in ventricles and increased filling pressure. If the hemodynamic of CS are not corrected, then it ultimately escalates to reduced tissue perfusion and multi-organ failure, thus there is an emphasis on early intervention to reduce mortality in these cases (Reynolds and Hochman, 2008).

For patients who are referred for high-risk PCI or cardiogenic shock, there are four treatment objectives taking care of the following mechanisms:

- Circulatory support (systemic perfusion)

- Ventricular support (LV unloading)

- Coronary perfusion

- Decongestion

Circulatory support is the improvement of mean arterial pressure, coronary perfusion means increase in microvascular coronary blood flow, and decongestion is defined as reduced total blood volume and elevated venous filling pressures (Esposito and Kapur, 2017). Left ventricular unloading, defined as reduction in myocardial work and wall stress, is the main target therapy of the MCs devices and it can be achieved by either reducing the pre-load (volume) or afterload (pressure), which reduces the myocardial oxygen demand (Burkhoff and Naidu, 2012). Pharmacologic agents, in unison, fail to correct this hemodynamic situation as the use of vasopressors and inotropes might improve mean arterial pressure but they either do not improve tissue perfusion or increase the myocardial work (Esposito and Kapur, 2017).

This is where the timely and righteous use of MCS devices comes into the picture. The devices can be pulsatile (IABP), or continuous flow pumps like axial flow pumps (Impella) or centrifugal flow pumps (VA-ECMO). The trans-valvular axial-flow pumps directly solve three of the four major objectives in the hemodynamic support equation by increasing mean arterial pressure, reducing LV pressure and volume, and increasing coronary blood flow (Esposito and Kapur, 2017). Decongestion, however, is not handled by the MCS devices and might require diuretic therapy or renal replacement therapy. Like their mechanism of action, the three devices have distinct hemodynamic profiles too; IABP reduces the pressure, Impella reduces both pressure and volume, and VA-ECMO increases the pressure. For this reason, the Impella pump is often deployed as an LV unloading strategy in VA-ECMO patients, resulting in improved patient hemodynamics (Patel et al., 2019). More details about the role of various MCS devices in improving the hemodynamic situation of the CS is given in Table 3.

\section{Indication of Use of Various MCS Devices}

The hemodynamics can give a cardiac surgeon a good idea about which MCS device will work better than the other in the given situation. If along with refractory CS there is severe aortic insufficiency, then Impella or LVAD might be a better option, while if there is hypoxemia along with ventricular failure then VA-ECMO along with LV venting should be done. It should also be considered if the failure is left ventricle dominant or right ventricle dominant. If the left ventricle is failing, then there is little use of using IABP, also VA-ECMO alone in this condition will further increase the workload of the heart. Appropriate drugs should also 
TABLE 3

The roles, indications, advantages, and disadvantages of various MCS devices

\begin{tabular}{|c|c|c|c|}
\hline Characteristic & IABP & VA-ECMO & Impella \\
\hline $\begin{array}{l}\text { Role in providing } \\
\text { Circulatory support } \\
\text { (systemic perfusion) }\end{array}$ & $\begin{array}{l}\text { Low support-if LV is dysfunctional then } \\
\text { IABP will not work properly }\end{array}$ & $\begin{array}{l}\text { Good support-increases mean arterial } \\
\text { pressure and maintain end-organ } \\
\text { perfusion }\end{array}$ & $\begin{array}{l}\text { Good support-increases } \\
\text { mean aortic root pressure } \\
\text { leading to improvement of } \\
\text { systemic perfusion }\end{array}$ \\
\hline $\begin{array}{l}\text { Role in providing } \\
\text { Ventricular support (LV } \\
\text { unloading) }\end{array}$ & $\begin{array}{l}\text { Some support-Reduces LV afterload } \\
\text { and increases LV cardiac output }\end{array}$ & $\begin{array}{l}\text { Negative role-Increases LV pressure, } \\
\text { wall stress and myocardial work }\end{array}$ & $\begin{array}{l}\text { Good support-Reduces both } \\
\text { LV pressure and volume }\end{array}$ \\
\hline $\begin{array}{l}\text { Role in coronary } \\
\text { perfusion }\end{array}$ & $\begin{array}{l}\text { Increases the diastolic pressure in the } \\
\text { aortic root and enhances the coronary } \\
\text { blood flow }\end{array}$ & $\begin{array}{l}\text { Decreases the heart rate so has some } \\
\text { role in reducing the myocardial } \\
\text { oxygen demand }\end{array}$ & $\begin{array}{l}\text { Increases the trans- } \\
\text { myocardial perfusion } \\
\text { gradient (aortic diastolic } \\
\text { pressure-LV } \\
\text { diastolic pressure), leading } \\
\text { to improved coronary } \\
\text { perfusion }\end{array}$ \\
\hline Role in decongestion & No role & No role & No role \\
\hline $\begin{array}{l}\text { Effect on pulmonary } \\
\text { circulation }\end{array}$ & $\begin{array}{l}\text { No significant effect on pulmonary } \\
\text { congestion (Haberkorn et al., 2020) }\end{array}$ & $\begin{array}{l}\text { Decreases the pulmonary artery } \\
\text { pressure and places the lungs at an } \\
\text { acutely elevated risk for ischemia and } \\
\text { pulmonary edema (Lamy et al., 1975) }\end{array}$ & $\begin{array}{l}\text { Maintains the lung } \\
\text { perfusion }\end{array}$ \\
\hline $\begin{array}{l}\text { Preferred type } \\
\text { of patients }\end{array}$ & $\begin{array}{l}\text { Preserved LVEF, MV-CAD, } \\
\text { Aortic Stenosis, Mitral Regurgitation }\end{array}$ & $\begin{array}{l}\text { Profound hypoxemia, cardiac arrest, } \\
\text { sepsis, multi-organ failure }\end{array}$ & $\begin{array}{l}\text { Cardiogenic shock, AMI/ } \\
\text { Shock, High Risk PCI with } \\
\text { Low EF }\end{array}$ \\
\hline Role in HF-CS & $\begin{array}{l}\text { No large, randomized studies have } \\
\text { evaluated the utility of IABP therapy in } \\
\text { HF-CS }\end{array}$ & $\begin{array}{l}\text { Some role in cardiogenic refractory } \\
\text { shock }\end{array}$ & $\begin{array}{l}\text { Major role in refractory } \\
\text { cardiogenic shock (Gaudard } \\
\text { et al., 2015) }\end{array}$ \\
\hline Major advantages & $\begin{array}{l}\text { Reduces in-hospital events (not } \\
\text { mortality) like dialysis, new onset of } \\
\text { stroke, pneumonia, sepsis (Mao et al., } \\
\text { 2016) }\end{array}$ & $\begin{array}{l}\text { Feasibility of implantation, easy access, } \\
\text { cost-effectiveness, can be used in } \\
\text { situations of circulatory arrest }\end{array}$ & $\begin{array}{l}\text { Fewer device related } \\
\text { complications, less invasive, } \\
\text { provide greater } \\
\text { hemodynamic support }\end{array}$ \\
\hline Major disadvantages & $\begin{array}{l}\text { Does not function properly in the } \\
\text { presence of an irregular ventricular } \\
\text { cardiac rhythm, pulselessness or cardiac } \\
\text { resuscitation, and contraindicated in } \\
\text { acute severe aortic insufficiency. } \\
\text { Some studies have associated its use } \\
\text { with a higher mortality (many patients } \\
\text { fail an IABP before they are put on other } \\
\text { devices leading to loss of critical time) } \\
\text { (Patel et al., 2014) }\end{array}$ & $\begin{array}{l}\text { Can cause severe side effects including } \\
\text { limb ischemia, } \\
\text { lower limb amputation, fasciotomy or } \\
\text { compartment syndrome, stroke, } \\
\text { pulmonary edema, and acute kidney } \\
\text { injury (Cheng et al., 2014) }\end{array}$ & $\begin{array}{l}\text { Bleeding complications, } \\
\text { cannot be used during valve } \\
\text { deployment procedures or } \\
\text { when TAVR device has to } \\
\text { be placed }\end{array}$ \\
\hline
\end{tabular}

Note: Source: Original.

use in managing the condition, especially the fourth mechanism, i.e., decongestion. Indications of use of various MCS devices, along with their major advantages and disadvantages, are further elaborated in Table 3. The prioritization of devices according to the patients and their indications is given in a flow chart in Fig. 4.

\section{Impella Technique: Case Studies}

Various studies have been done to assess the outcomes and complications of using the Impella device. A study done by Ouweneel et al. (2019) has recorded 12 years of clinical experience with Impella in patients with cardiogenic shock, after acute myocardial infarction. This registry concluded that Impella treatment in cardiogenic shock after acute myocardial infarction is feasible, although mortality rates remain high, and complications occur. Lauten et al. (2013) studied the evaluation of safety and efficacy of the Impella2.5-percutaneous left-VAD in patients with cardiogenic shock after acute myocardial infarction. They also observed a high 30-day mortality, however, the lactate levels improved suggesting better organ perfusion. A study by Lemaire et al. (2014) showed improved results as compared to earlier studies. The authors suggested that 30day mortality and complication rates were within acceptable levels.

After a careful evaluation of the data collected from the global catheter-based ventricular assist device (cVAD) 


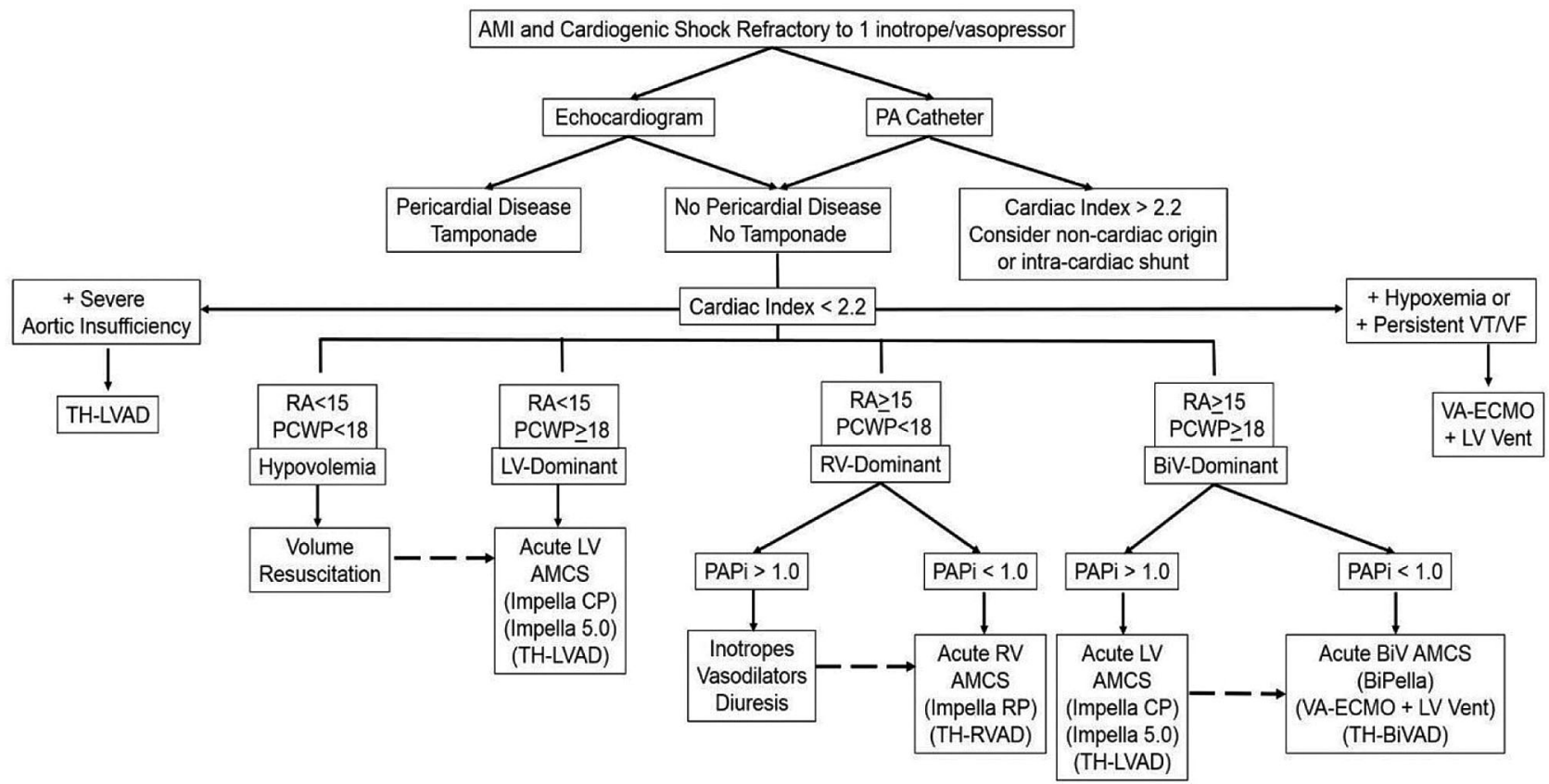

FIGURE 4. The cardiogenic shock algorithm and prioritization of MCS devices according to patients' hemodynamic condition (Reproduced from: Esposito and Kapur (2017)).

registry, Elkayam et al. (2019) concluded that MCS with Impella devices can be successfully used as a bridge to early improvement, heart recovery, or successful implantation of durable LVAD in women with peripartum cardiomyopathy (PPCM) complicated by severe LV dysfunction. Another data suggests that temporary use of the Impella CP device could be a useful therapeutic option that can be tried as a bridge to recovery, not only with ischaemic but also with non-ischaemic cardiogenic shock patients (Maniuc et al., 2019). Nersesian et al. (2019) studied patients who received support from a left ventricular assist device (Impella), while LevitronixCentri Mag was used for right ventricular support. Survival after MCS implantation for left as well as right heart failure in cardiogenic shock remains low but is rather good in patients without being on mechanical support. This study concluded that short-term MCS remains an option of choice if right, left, or biventricular support is needed. O'Neill et al. (2014) evaluated the periprocedural characteristics and outcomes of patients supported with Impella 2.5 prior to percutaneous coronary intervention (pre-PCI) vs. those who received it after PCI (post-PCI) in the setting of cardiogenic shock (CS) complicating an acute myocardial infarction (AMI). This study analysed data from 154 consecutive patients who underwent PCI and Impella 2.5 support from 38 US hospitals and it was noted that early initiation of hemodynamic support prior to PCI with Impella 2.5 is associated with more complete revascularization and improved survival in the setting of refractory CS complicating an AMI. van Dort et al. (2020) conducted a meta-analysis in 2019 , in which, haemodynamic effects of the Impella device in a clinical setting were investigated. The outcomes that were studied were cardiac power (CP), cardiac power index (CPI), survival rates and other haemodynamic data. The analysis included 12 studies of moderate quality, with a total of 596 patients. This study suggests that in patients with cardiogenic shock, Impella support seems effective in augmenting $\mathrm{CP}(\mathrm{I})$. A systematic review and meta-analysis conducted by Hill et al. (2019) deduced that Impella devices were associated with good survival and low rates of complications and safety outcomes across all combinations of indication and study types analyzed. The review demonstrates very encouraging survival in cardiogenic shock patients and very good 30-day outcomes in patients undergoing prophylactic support for high-risk PCI.

A few studies have compared the performance of Impella with other devices. Alushi et al. (2019) conducted a singlecentre, retrospective study, which included patients with AMICS, receiving pMCS (percutaneous mechanical support) with either Impella or IABP. A total of 116 target patients were observed out of which, $62(53 \%)$ received Impella and 54 (47\%) IABP. They observed that despite similar baseline mortality risk (IABP-SHOCK II high risk score of $18 \%$ vs. 20\%; $P=0.76)$, Impella treatment significantly reduced the inotropic score $(P<0.001)$, lactate levels $(P<0.001)$ and SAPS II $(P=0.02)$ and improved left ventricular ejection fraction $(P=0.01)$. However, all-cause mortality at 30 days was similar with Impella and IABP $(52 \%$ and $67 \%$, respectively; $P=0.13$ ), but bleeding complications were seen more frequent in the Impella group (3vs. 4 units of transfused erythrocytes concentrates; $P=0.03$ ). The study concluded that the haemodynamic support (in patients with AMICS) with the Impella device had no significant effect on 30-day mortality, as compared with IABP and large randomised trials are needed to find out the effect of Impella on the outcome. We further found two small randomized controlled trials comparing Impella and IABP for patients with cardiogenic shock (Anderson et al., 2015; Ouweneel et al., 2017b; Seyfarth et al., 2008). One study randomized patients with $\mathrm{AMI}$ and cardiogenic shock to Impella $2.5(\mathrm{~N}=12) v s$. IABP counterpulsation $(\mathrm{N}=13)$. Compared with IABP, the Impella group had higher cardiac index $\left(0.49 \mathrm{~L} / \mathrm{min} / \mathrm{m}^{2}\right.$ vs. $\left.0.11 \mathrm{~L} / \mathrm{min} / \mathrm{m}^{2} ; P=0.02\right)$ at 
30 min after implantation, but 30-day mortality was roughly $50 \%$ in each group $(P=0.97)$ (Seyfarth et al., 2008). More recently, Impella CP was compared with IABP in 48 patients with shock after AMI. Thirty-day mortality was about $50 \%$ in each group $(P=0.92)$ (Gaudard et al., 2015). Neither study was powered to detect differences in mortality. These trial data were combined with data from a study of Impella $2.5 \mathrm{vs}$. IABP in 21 subjects with cardiogenic pre-shock (Ouweneel et al., 2015). The resultant metaanalysis reported no difference in mortality at 30 days (RR 0.99 , [CI $0.62-1.58$ ]; $P=0.95$ ) or 6 months (RR 1.15, [0.741.48]; $P=0.53$ ) (Ouweneel et al., 2017a). A meta-analysis also suggests that neither LVADs nor IABP improves short or long-term survival in hr-PCI patients, though, LVADs are more likely to reduce repeat revascularization after PCI (Shi et al., 2019). An animal study, however, has suggested that haemodynamics and blood flow to the brain and kidneys were significantly better on Impella support, suggesting that the Impella is superior to the IABP in a state of ischaemia induced left ventricular failure (MøllerHelgestad et al., 2015).

The evidence for the usefulness of ECMO in patients with cardiogenic shock is scant and there are no randomized controlled studies comparing ECMO with pVADs (Miller et al., 2017). Few observational studies are available in the literature though. Garan et al. (2019) did a prospective comparison of a Percutaneous Ventricular Assist Device (pVAD) and Venoarterial Extracorporeal Membrane Oxygenation (VA-ECMO) for patients with cardiogenic shock following acute myocardial infarction. In total, 51 patients received VA-ECMO or pVAD following AMI; 20 received VA-ECMO, and 31 received $\mathrm{PVAD}$. Patients treated with pVAD or VA-ECMO were similar in baseline characteristics at initial device insertion except that the latter were on more vasopressors and were more likely to have an intra-aortic balloon pump. Survival at 1 and 2 years did not differ significantly between device groups $(P=0.42)$. This study concluded that following AMI-related CS, pVAD- and VA-ECMO-treated patients had similar outcomes and a randomized trial comparing these 2 therapies may be warranted. A recent meta-analysis of cohort studies found that patients treated with ECMO had a higher 30-day survival compared with IABP $(P<0.001$, NNT 13), but no difference when compared with pVADs $(P=0.70)$ (Ouweneel et al., 2016). Another retrospective case series reported lower 30-day mortality in patients with cardiogenic shock undergoing ECMO-assisted PCI $v$ s. ECMO-unassisted PCI $(39.1 \%$ vs. $72 \%$, respectively). However, the ECMO-unassisted group was a historic control group (1993-2002) and likely received a different standard of care (Sheu et al., 2010). Another study concludes that percutaneous left ventricular assist devices such as TandemHeart and Impella are easier to institute than ECMO and are better for hemodynamics compared with the IABP but also have not yet shown a mortality benefit (Khan et al., 2014). In another study it was noted that though the 30-day mortality did not differ significantly between the Impella and ECMO group, but patients with Impella support had fewer device-related complications than patients treated with ECMO (respectively, 17\% vs. 40\%) (Karami et al., 2020).

It can be said that MCS device use during PCI should be individualized based on several factors with a recommended use in patients with the greatest potential benefit and a relatively low risk of device-related complications (Asleh and Resar, 2019). Impella cardiac axial pump can stabilize the haemodynamics of patients with cardiogenic shock. It can significantly reduce the load on the left ventricle, improve effective blood perfusion and accelerate the recovery of heart function. Therefore, it effectively reduces the hospital mortality of patients with cardiogenic shock and improves the prognosis of patients. Therefore, for patients with cardiogenic shock, early diagnosis, early revascularization, and timely treatment of Impella system are recommended. The combined use of MCS devices is also common. A meta-analysis from six randomized controlled trials and 24 observational studies totalling 15799 patients reported, relative to comparisons of ECMO plus IABP $v s$. IABP or ECMO plus IABP vs. ECMO, a significant protective effect of ECMO plus IABP on in-hospital mortality compared to IABP or ECMO used alone (Romeo et al., 2016). Additionally, reactive unloading with the help of Impella device while using ECMO was reported to be a viable strategy by provides better than anticipated survival amongst patients (Piechura et al., 2020). There is also an increasing evidence of a possible timing issue favouring early application of MCS (Jensen et al., 2018; O'Neill et al., 2018; Huang et al., 2018).

Though the debate on the merits and demerits of the MCS devices is never ending, short term clinical results are encouraging, and these devices have provided a ray of hope for the condition that has skyrocketing mortality rates. The current literature suggests that the outcomes of use of Impella and other mechanical circulatory support devices like IABP and VA-ECMO are comparable, and large and sufficiently powered studies are needed to establish the superiority of one over the other.

\section{Way Ahead-Future Directions and Innovations Needed}

Instead of considering these devices as competing technologies, a deep understanding should be developed of their mechanisms and functioning, and they should be used in combination, wherever indicated, for improving the prognosis of patients. Their advantages and disadvantages should be used to innovate new devices that promise better outcomes. Research should also be escalated as far as the current devices are concerned as there is a lack of large, multi-centric, randomised controlled trials that can establish the superiority of use of one device over another in different clinical situations. The use of MCS devices in various situations like among elderly patients with multicomorbidities, undergoing procedures such as transcatheter aortic valve replacement (TAVR) is also underexplored. It is also imperative to identify age-based trends in the outcomes and of circulatory support (MCS) use among patients with both AMI and non-AMI associated shock. Apart from the type of device, the timing of device use has also received some attention in recent years. Hemodynamic data (like 
cardiac power output, cardiac filling pressures, transpulmonary gradient, etc.) should be taken into consideration while making acute MCS decisions as the condition of a patient in CS escalates rapidly to a point where MCS devices fail to improve their condition. Rapid and accurate measurement of these parameters can guide a cardiac surgeon about the possibility of which device might do better than the other. Lastly, more research is needed on the bio-trauma or the shear forces that these pumps/devices cause on the blood cells or circulation.

Availability of Data and Materials: Data sharing not applicable to this article as no datasets were generated or analyzed during the current study.

Author Contribution: Dr. Rafiq Ahmed Bhat contributed in writing the first draft of the manuscript. Dr. Syed Manzoor Ali contributed in the drawing of the tables. Dr. Yoosuf Ali Ashraf Muhammad Hussenbocus contributed in editing the draft and together with Dr. Javaid Akhter Bhat and Dr. Abdul Aleem Khan provided critical inputs in the review. Dr. Akanksha Rathi, Dr. Syed Maqbool, Dr. Raja Saqib Iqbal, Dr. Md Monowarul Islam, Dr. Yongsheng Qu, Dr. You Zhang, Dr. Yuxiao Sun, Dr. Wentao Xiao, Dr. Abhishek Tibrewal contributed in the collection of the literature Dr. Chuanyu Gao finalized the manuscript.

Funding Statement: The authors received no specific funding for this study.

Conflicts of Interest: The authors declare that they have no conflicts of interest to report regarding the present study.

\section{References}

Alushi B, Douedari A, Froehlig G, Knie W, Wurster TH et al. (2019). Impella $v s$. IABP in acute myocardial infarction complicated by cardiogenic shock. Open Heart 6: e000987. DOI 10.1136/ openhrt-2018-000987.

Anderson MB, Goldstein J, Milano C, Morris LD, Kormos RL et al. (2015). Benefits of a novel percutaneous ventricular assist device for right heart failure: The prospective RECOVER RIGHT study of the Impella RP device. Journal of Heart and Lung Transplantation 34: 1549-1560. DOI 10.1016/j. healun.2015.08.018.

Asleh R, Resar JR (2019). Utilization of percutaneous mechanical circulatory support devices in cardiogenic shock complicating acute myocardial infarction and high-risk percutaneous coronary interventions. Journal of Clinical Medicine 8: 1209. DOI $10.3390 / \mathrm{jcm} 8081209$.

Bernhardt AM, Potapov E, Schibilsky D, Ruhparwar A, Schmitto JD et al. (2020). First in men multicenter experience with the Impella 5.5. Journal of Heart and Lung Transplantation 39: S99. DOI 10.1016/j.healun.2020.01.950.

Burkhoff D, Naidu SS (2012). The science behind percutaneous hemodynamic support: A review and comparison of support strategies. Catheterization and Cardiovascular Interventions 80: 816-829. DOI 10.1002/ccd.24421.

Cardozo S, Ahmed T, Belgrave K (2015). Impella induced massive hemolysis: Reemphasizing echocardiographic guidance for correct placement. Case Reports in Cardiology 2015: 1-3. DOI $10.1155 / 2015 / 464135$.
Cheng R, Hachamovitch R, Kittleson M, Patel J, Arabia F et al. (2014). Complications of extracorporeal membrane oxygenation for treatment of cardiogenic shock and cardiac arrest: A metaanalysis of 1,866 adult patients. Annals of Thoracic Surgery 97: 610-616. DOI 10.1016/j.athoracsur.2013.09.008.

Elkayam U, Schäfer A, Chieffo A, Lansky A, Hall S et al. (2019). Use of Impella heart pump for management of women with peripartum cardiogenic shock. Clinical Cardiology 42: 974981. DOI 10.1002/clc.23249.

Esposito ML, Kapur NK (2017). Acute mechanical circulatory support for cardiogenic shock: The door to support time. F1000Research 6: 737. DOI 10.12688/f1000research.

Garan AR, Takeda K, Salna M, Vandenberge J, Doshi D et al. (2019). Prospective comparison of a percutaneous ventricular assist device and venoarterial extracorporeal membrane oxygenation for patients with cardiogenic shock following acute myocardial infarction. Journal of the American Heart Association 8: e012171. DOI 10.1161/JAHA.119.012171.

Gaudard P, Mourad M, Eliet J, Zeroual N, Culas G et al. (2015). Management and outcome of patients supported with Impella 5.0 for refractory cardiogenic shock. Critical Care 19: 1787. DOI 10.1186/s13054-015-1073-8.

Haberkorn S, Uwarow A, Haurand J, Jung C, Kelm M et al. (2020). Percutaneous left ventricular assist support is associated with less pulmonary congestion and lower rate of pneumonia in patients with cardiogenic shock. Open Heart 7: e001385. DOI 10.1136/openhrt-2020-001385.

Hajjar LA, Teboul JL (2019). Mechanical circulatory support devices for cardiogenic shock: State of the art. Critical Care 23: 760. DOI 10.1186/s13054-019-2368-y.

Health Jade Team (2019). Impella Device. https://healthjade.net/ impella-device/.

Hill J, Banning A, Burzotta F, Chieffo A, Schieffer B et al. (2019). A systematic literature review and meta-analysis of Impella devices used in cardiogenic shock and high risk percutaneous coronary interventions. Interventional Cardiology 11: 161-171. DOI 10.4172/Interventional-Cardiology.1000624.

Huang CC, Hsu JC, Wu YW, Ke SR, Huang JH et al. (2018). Implementation of extracorporeal membrane oxygenation before primary percutaneous coronary intervention may improve the survival of patients with ST-segment elevation myocardial infarction and refractory cardiogenic shock. International Journal of Cardiology 269: 45-50. DOI 10.1016/j.ijcard.2018.07.023.

Ibanez B, James S, Agewall S, Antunes MJ, Bucciarelli-Ducci C et al. (2018). 2017 ESC guidelines for the management of acute myocardial infarction in patients presenting with STsegment elevation: The Task Force for the management of acute myocardial infarction in patients presenting with STsegment elevation of the European Society of Cardiology (ESC). European Heart Journal 39: 119-177. DOI 10.1093/ eurheartj/ehx393.

Jensen PB, Kann SH, Veien KT, Møller-Helgestad OK, Dahl JS et al. (2018). Single-centre experience with the Impella $\mathrm{CP}, 5.0$ and RP in 109 consecutive patients with profound cardiogenic shock. European Heart Journal: Acute Cardiovascular Care 7: 53-61.

Johannsen L, Mahabadi AA, Totzeck M, Krueger A, Jánosi RA et al. (2019). Access site complications following Impellasupported high-risk percutaneous coronary interventions. Scientific Reports 9: 17844.

Kapur NK, Paruchuri V, Majithia A, Esposito M, Shih H et al. (2015). Hemodynamic effects of standard vs. larger-capacity 
intraaortic balloon counterpulsation pumps. Journal of Invasive Cardiology 27: 182-188.

Karami M, den Uil CA, Ouweneel DM, Scholte NT, Engström AE et al. (2020). Mechanical circulatory support in cardiogenic shock from acute myocardial infarction: Impella $\mathrm{CP} / 5.0$ vs. ECMO. European Heart Journal: Acute Cardiovascular Care 9: 164-172.

Khan MH, Corbett BJ, Hollenberg SM (2014). Mechanical circulatory support in acute cardiogenic shock. F1000Prime Reports 6: 91. DOI 10.12703/P6-91.

Khan TM, Siddiqui AH (2021). Intra-Aortic Balloon Pump. In: StatPearls. Treasure Island (FL): StatPearls Publishing; https://www.ncbi.nlm.nih.gov/books/NBK542233/\#_NBK542233_ pubdet.

Lamy M, Eberhart RC, Fallat RJ, Dietrich HP, Ratliff J et al. (1975). Effects of extracorporeal membrane oxygenation (ECMO) on pulmonary hemodynamics, gas exchange and prognose. Transactions-American Society for Artificial Internal Organs 21: 188-198.

Lauten A, Engström AE, Jung C, Empen K, Erne P et al. (2013). Percutaneous left-ventricular support with the Impella-2.5assist device in acute cardiogenic shock: Results of the ImpellaEUROSHOCK-registry. Circulation: Heart Failure 6: 23-30.

Lemaire A, Anderson MB, Lee LY, Scholz P, Prendergast T et al. (2014). The Impella device for acute mechanical circulatory support in patients in cardiogenic shock. Annals of Thoracic Surgery 97: 133-138. DOI 10.1016/j.athoracsur.2013.07.053.

Mandawat A, Rao SV (2017). Percutaneous mechanical circulatory support devices in cardiogenic shock. Circulation: Cardiovascular Interventions 10: e004337.

Maniuc O, Salinger T, Anders F, Müntze J, Liu D et al. (2019). Impella $\mathrm{CP}$ use in patients with non-ischaemic cardiogenic shock. ESC Heart Failure 6: 863-866. DOI 10.1002/ehf2.12446.

Mao CT, Wang JL, Chen DY, Tsai ML, Lin YS et al. (2016). Benefits of intraaortic balloon support for myocardial infarction patients in severe cardiogenic shock undergoing coronary revascularization. PLoS One 11: e0160070. DOI 10.1371/ journal.pone.0160070.

Mebazaa A, Combes A, Van Diepen S, Hollinger A, Katz JN et al. (2018). Management of cardiogenic shock complicating myocardial infarction. Intensive Care Medicine 44: 760-773. DOI 10.1007/s00134-018-5214-9.

Miller PE, Solomon MA, McAreavey D (2017). Advanced percutaneous mechanical circulatory support devices for cardiogenic shock. Critical Care Medicine 45: 1922-1929. DOI 10.1097/CCM.0000000000002676.

Møller-Helgestad OK, Poulsen CB, Christiansen EH, Lassen JF, Ravn HB (2015). Support with intra-aortic balloon pump vs. Impella2. $5^{\circ}$ and blood flow to the heart, brain and kidneys -An experimental porcine model of ischaemic heart failure. International Journal of Cardiology 178: 153-158. DOI 10.1016/j.ijcard.2014.10.153.

Nersesian G, Hennig F, Müller M, Mulzer J, Tsyganenko D et al. (2019). Temporary mechanical circulatory support for refractory heart failure: The German Heart Center Berlin experience. Annals of Cardiothoracic Surgery 8: 76-83. DOI 10.21037/acs.2018.12.01.

O’Neill WW, Grines C, Schreiber T, Moses J, Maini B et al. (2018). Analysis of outcomes for 15,259 US patients with acute myocardial infarction cardiogenic shock (AMICS) supported with the Impella device. American Heart Journal 202: 33-38. DOI 10.1016/j.ahj.2018.03.024.
O’Neill WW, Schreiber T, Wohns DH, Rihal C, Naidu SS et al. (2014). The current use of Impella 2.5 in acute myocardial infarction complicated by cardiogenic shock: Results from the USpella registry. Journal of Interventional Cardiology 27: 1-11. DOI 10.1111/joic.12080.

Ouweneel DM, de Brabander J, Karami M, Sjauw KD, Engström AE et al. (2019). Real-life use of left ventricular circulatory support with Impella in cardiogenic shock after acute myocardial infarction: 12 years AMC experience. European Heart Journal: Acute Cardiovascular Care 8: 338-349.

Ouweneel DM, Engstrom AE, Sjauw KD, Hirsch A, Hill JM et al. (2015). Experience from a randomized controlled trial with Impella 2.5 vs. IABP in STEMI patients with cardiogenic pre-shock. Lessons learned from the IMPRESS in STEMI trial. International Journal of Cardiology 202: 894-896. DOI 10.1016/j.ijcard.2015.10.063.

Ouweneel DM, Eriksen E, Seyfarth M, Henriques JP (2017a). Percutaneous mechanical circulatory support vs. intraaortic balloon pump for treating cardiogenic shock: Metaanalysis. Journal of the American College of Cardiology 69: 358-360. DOI 10.1016/j.jacc.2016.10.026.

Ouweneel DM, Eriksen E, Sjauw KD, van Dongen IM, Hirsch A et al. (2017b). Percutaneous mechanical circulatory support $v s$. intra-aortic balloon pump in cardiogenic shock after acute myocardial infarction. Journal of the American College of Cardiology 69: 278-287. DOI 10.1016/j.jacc.2016.10.022.

Ouweneel DM, Schotborgh JV, Limpens J, Sjauw KD, Engström AE et al. (2016). Extracorporeal life support during cardiac arrest and cardiogenic shock: A systematic review and metaanalysis. Intensive Care Medicine 42: 1922-1934. DOI 10.1007/s00134-016-4536-8.

Patel H, Shivaraju A, Fonarow GC, Xie H, Gao W et al. (2014). Temporal trends in the use of intraaortic balloon pump associated with percutaneous coronary intervention in the United States, 1998-2008. American Heart Journal 168: 363-373.e12. DOI 10.1016/j.ahj.2014.02.015.

Patel MR, Smalling RW, Thiele H, Barnhart HX, Zhou Y et al. (2011). Intra-aortic balloon counterpulsation and infarct size in patients with acute anterior myocardial infarction without shock: The CRISP AMI randomized trial. JAMA 306: 1329-1337. DOI 10.1001/jama.2011.1280.

Patel SM, Lipinski J, Al-Kindi SG, Patel T, Saric P et al. (2019). Simultaneous venoarterial extracorporeal membrane oxygenation and percutaneous left ventricular decompression therapy with Impella is associated with improved outcomes in refractory cardiogenic shock. ASAIO Journal 65: 21-28. DOI 10.1097/MAT.0000000000000767.

Piechura LM, Coppolino A, Mody GN, Rinewalt DE, Keshk M et al. (2020). Left ventricle unloading strategies in ECMO: A single-center experience. Journal of Cardiac Surgery 35: 1514-1524. DOI 10.1111/jocs.14644.

Reynolds HR, Hochman JS (2008). Cardiogenic shock: Current concepts and improving outcomes. Circulation 117: 686697. DOI 10.1161/CIRCULATIONAHA.106.613596.

Romeo F, Acconcia MC, Sergi D, Romeo A, Francioni S et al. (2016). Percutaneous assist devices in acute myocardial infarction with cardiogenic shock: Review, meta-analysis. World Journal of Cardiology 8: 98-111. DOI 10.4330/wjc.v8.i1.98.

Seyfarth M, Sibbing D, Bauer I, Fröhlich G, Bott-Flügel L et al. (2008). A randomized clinical trial to evaluate the safety and efficacy of a percutaneous left ventricular assist device vs. intra-aortic balloon pumping for treatment of cardiogenic shock caused by myocardial infarction. 
Journal of the American College of Cardiology 52: 15841588. DOI 10.1016/j.jacc.2008.05.065.

Sheu JJ, Tsai TH, Lee FY, Fang HY, Sun CK et al. (2010). Early extracorporeal membrane oxygenator-assisted primary percutaneous coronary intervention improved 30-day clinical outcomes in patients with ST-segment elevation myocardial infarction complicated with profound cardiogenic shock. Critical Care Medicine 38: 1810-1817. DOI 10.1097/CCM.0b013e3181e8acf7.

Shi W, Wang W, Wang K, Huang W (2019). Percutaneous mechanical circulatory support devices in high-risk patients undergoing percutaneous coronary intervention: A metaanalysis of randomized trials. Medicine 98: e17107. DOI 10.1097/MD.0000000000017107.
Sintek MA, Gdowski M, Lindman BR, Nassif M, Lavine KJ et al. (2015). Intra-aortic balloon counterpulsation in patients with chronic heart failure and cardiogenic shock: Clinical response and predictors of stabilization. Journal of Cardiac Failure 21: 868876. DOI 10.1016/j.cardfail.2015.06.383.

van Diepen S, Katz JN, Albert NM, Henry TD, Jacobs AK et al. (2017). Contemporary management of cardiogenic shock: A scientific statement from the American Heart Association. Circulation 136: 231-268. DOI 10.1161/CIR.0000000000000525.

van Dort DIM, Peij KR, Manintveld OC, Hoeks SE, Morshuis WJ et al. (2020). Haemodynamic efficacy of microaxial left ventricular assist device in cardiogenic shock: A systematic review and meta-analysis. Netherlands Heart Journal 28: 179-189. DOI 10.1007/s12471-019-01351-7. 